\title{
Oncology providers' perspectives on endocrine therapy prescribing and management
}

\author{
This article was published in the following Dove Press journal: \\ Patient Preference and Adherence \\ 30 September 2016 \\ Number of times this article has been viewed
}

\author{
Stephanie B Wheeler ${ }^{1,2}$ \\ Megan C Roberts' \\ Diane Bloom' \\ Katherine E Reeder-Hayes ${ }^{2,3}$ \\ Maya Espada' \\ Jeffrey Peppercorn ${ }^{4}$ \\ Carol E Golin ${ }^{5,6}$ \\ Jo Anne Earp ${ }^{2,5}$ \\ 'Department of Health Policy \\ and Management, ${ }^{2}$ Lineberger \\ Comprehensive Cancer Center, \\ ${ }^{3}$ Division of Hematology and \\ Oncology, University of North \\ Carolina at Chapel Hill, Chapel \\ Hill, NC, ${ }^{4}$ Division of Hematology/ \\ Oncology, Massachusetts General \\ Hospital, Boston, MA, ${ }^{5}$ Department \\ of Health Behavior, ${ }^{6}$ Department \\ of Medicine, University of \\ North Carolina at Chapel Hill, \\ Chapel Hill, NC, USA
}

Purpose: Adjuvant endocrine therapy (ET) can reduce the risk of recurrence among females with hormone receptor-positive breast cancer. Overall, initiation and adherence to ET are suboptimal, though reasons are not well described. The study's objective was to better understand ET decision making, prescribing, and patient management from oncology providers' perspectives.

Methods: Using purposive sampling, we recruited oncology providers who saw five or more breast cancer patients per week $(n=20)$. We conducted 30-45-minute telephone interviews, using a semistructured guide to elicit perspectives on ET use. We used thematic content analysis to systematically identify categories of meaning and double-coded transcripts using Atlas.ti.

Results: Providers recommend ET to all eligible patients except those with contraindications or other risk factors. Providers base their ET prescribing decisions on the patient's menopausal status, side effects, and comorbidities. ET is typically discussed multiple times: at the onset of breast cancer treatment and in more detail after other treatment completion. Providers felt that the associated recurrence risk reduction is the most compelling argument for patients during ET decision making. While providers rarely perceived noninitiation as a problem, nonadherence was prevalent, often due to unresolvable side effects.

Conclusion: From the clinicians' perspectives, side effects from ET are the dominant factor in nonadherence. Efforts to improve adherence should focus on strategies to minimize side effects and ensure clinicians and patients are well informed regarding optimal side effect management. This finding has important implications for novel endocrine regimens that offer improved outcomes through longer duration or more intensive therapy.

Keywords: breast cancer, endocrine therapy, oncology, oncologist, qualitative interviews

\section{Introduction}

Endocrine therapy (ET) is an important part of treatment for females with hormone receptor-positive $(\mathrm{HR}+)$ breast cancers. Both forms of ET, tamoxifen and aromatase inhibitors (AI), have been shown to reduce in-breast recurrence by $\sim 40 \%$ and breast cancer mortality by a third when taken as recommended for at least 5 years. ${ }^{1}$ Unfortunately, up to half of clinically eligible females fail to initiate ET and of those who do initiate, approximately a quarter are nonadherent and another quarter have discontinued by 5 years. ${ }^{2}$ The reasons for underuse of ET are multifaceted and likely driven by oncology providers' prescribing and management patterns, as well as individual patients' motivation and self-efficacy to continue taking an oral medication that is known to cause burdensome side effects. ${ }^{3-5}$ The most commonly reported side effects to ET are hot flashes and night sweats (associated primarily with tamoxifen), bone loss, and joint and muscle pain (associated primarily with AIs). ${ }^{6}$

In a systematic review, interpersonal characteristics associated with optimal ET initiation and adherence included: provider referral and recommendation, quality
Correspondence: Stephanie B Wheeler Department of Health Policy and Management, University of North Carolina at Chapel Hill, I35 Dauer Drive, CB74I I, Chapel Hill, NC 275I6, USA

$\mathrm{Tel}+\mathrm{I} 9199667374$

Fax + I 9198436362

Email stephanie_wheeler@unc.edu 
of patient-provider communication, and social support. ${ }^{2}$ Patients who reported seeing a medical oncologist, receiving patient-centered care, valuing their provider's opinion, having high self-efficacy in communications with their provider(s), and receiving enough information about ET from their provider(s) were more likely to be adherent and persistent with ET, whereas other factors commonly assumed to be related to medication nonadherence, such as frequency of office visits, patient education and financial stability, urban/ rural residence, and size of the treating facility, were not significant predictors of ET use. ${ }^{2}$ These findings suggest that the quality of the patient-provider relationship may provide clues as to whether patients will take ET as prescribed for the full duration of treatment, and how providers can best support patients during this phase of care.

Oncology care providers play an important role in prescribing ET, preparing patients for side effects and other difficulties they may encounter while taking ET, and managing side effects and medication changes over time. They also play a part in directing patients to resources for support and counseling about ET, motivating patients to continue taking ET as prescribed, and communicating with patients and their primary care providers about the appropriate duration and continued management of ET use. ${ }^{7,8}$ With new evidence suggesting that longer duration of ET or more intensive therapy may be warranted in some cases, ${ }^{9,10}$ more attention should be paid to oncology providers' roles in optimizing ET use to prevent breast cancer recurrence, ensure high quality of life, and reduce breast cancer-related death. In this study, we sought to contribute to the existing literature on ET use by soliciting oncology providers' perspectives on the prescribing and management of ET in their breast cancer patient populations. Rarely has the provider's voice been incorporated in studies focused upon oral oncology medication adherence. However, adding these important players' perspectives to the ET discussion may provide insight into the processes of care around ET initiation and longer term use, the realities and challenges associated with supporting patients taking ET, and strategies used by providers to prepare patients for - and help to manage - side effects in practice.

\section{Methods}

Twenty in-depth telephone interviews were conducted with oncologists, surgeons, physicians' assistants, and oncology nurses as part of a larger patient and provider qualitative research study on the barriers and facilitators to ET use. Using purposive sampling, we identified 20 oncology providers.
Most were identified through the North Carolina Oncology Association Web site (http://ncoa-northcarolina.com/) and through contacts with clinical partners. We emailed and faxed a recruitment flyer to potentially eligible providers, and interested providers contacted the study team in order to participate. Providers were eligible to participate in an interview if they saw five or more breast cancer patients per week. The majority of participants were based at two large academic medical centers and community-based practices across North Carolina.

Each interview lasted $\sim 30-60$ minutes and was conducted by a professional moderator using a semistructured discussion guide to allow for a deeper exploration of the topics under investigation. The overall objective of the provider interviews was to better understand providers' perceptions and experiences regarding prescribing and managing ET among their HR+ breast cancer patients. These interviews asked specific questions in order to: 1) Understand when and how providers first introduce the concept of ET to their patients; 2) Understand providers' perceptions about the best and most compelling approach for presenting the idea of ET to patients; 3) Learn more about how providers decide which ET to prescribe and for how long; 4) Assess how much of a problem providers perceive noninitiation to be among eligible patients and the reasons some refuse ET at the outset; 5) Assess how much of a problem nonadherence is among eligible patients and identify the reasons for nonadherence; and 6) Understand strategies employed by providers to support ET use.

All interviews were conducted by a professional interviewer (DB) and a note-taker (MCR), neither of whom was involved with patient management or care. The interviewer and note-taker were familiar with research about breast cancer, ET, and clinical guidelines for ET use among eligible patients. Interviews were digitally recorded, professionally transcribed, de-identified, and transferred to Atlas.ti for analysis. Members of the study team (SBW, MCR, KRH, and DB) first reviewed all transcripts and created an initial concept code list. We used thematic content analysis to identify categories of meaning. Response patterns, both within a particular question or concept and between concepts, were explored. These concept codes were systematically discussed and defined clearly, with exemplar codes as well as exclusions made explicit to facilitate coding and ensure consistent application of codes across transcripts and coders. Two authors (DB, MCR) then coded the first 25\% (five) of provider transcripts and discussed code application. Very few inconsistencies were identified in code application. 
Any inconsistencies or overarching questions about code meaning were discussed and reconciled by the research team. The codebook was revised where applicable. Once consensus was reached on the first five transcripts, the remaining transcripts were coded individually by a single coder following a common final codebook (Table 1).
As before, any questions about code meaning or application were addressed as they emerged with the second coder and lead investigator (SBW). This process ensured consistency, reliability, and reproducibility in our analytic coding process. We achieved saturation of themes before completing the 20 interviews.

Table I Final codebook descriptions and exemplary quotes

\begin{tabular}{ll}
\hline Code & Definition \\
\hline Age-based decision making & $\begin{array}{l}\text { Description of how age influences } \\
\text { prescribing behavior }\end{array}$
\end{tabular}

Exemplary quotes

"I think when you're dealing with the more elderly women, I think quality of life is a more prominent feature and wondering, not worrying so much about the quantity. But when you're dealing with younger women, it's much more focused on kind of getting rid of the cancer and keeping it gone forever. So, there's sort of a shift depending on the age and just temperament of the patient."

Beliefs in line with behavior

Comorbid conditions

Compelling arguments

Coping

Cost

Double-edged sword

Fear

Fertility

Information seeking
Discussion of how patients' beliefs are concordant/discordant with medication behavior

Discussion of the influence of comorbidities on medication behavior

Includes responses to "what is the most compelling argument for ET initiation?"

Includes management strategies for reported patient side effects

Discussion of cost as a barrier or facilitator for ET use

Discussion of risks balanced with benefits

Perceived patients' fears influence on medication behavior Fertility as contraindicated to ET use

Includes discussion of informational materials that providers use, including verbal, audio, and visual materials
"Some of those patients just refuse to take anything. Some of them are pretty happy with surgical therapy. You know, they have an operation, they cut it out, they think they're cured and no matter how much data you talk to them they just don't want to take a pill."

"So, earlier this month I saw a younger woman with DCIS. And you would normally consider tamoxifen but she has a history of recurrent venousthrombo-embolic disease and I felt the chemo-prevention was outweighed by the risks of thrombo-embolic disease."

"And so, I almost stand on top of my head to beg them, you know [...] And the things that l'll tell them is that sometimes you'll get an 85-, 90-year-old female to come in and there's cancer growing all up their chest and you can put them on an aromatase inhibitors if they're ER- or PR-positive. And that's not chemotherapy. And all the disease will go away within a few months' time. So, l'll give them these extreme examples to try to show 'this is how important this is.",

"Well, with tamoxifen I try to stay away from any of the [antidepressants] that can have interference with tamoxifen metabolism. So, I tend to select Lexapro Effexor sometimes if the issue is more hot flashes than mood disorder. Then Effexor can be helpful. [...] And then some folks just have hot flashes primarily at nighttime. Sometimes we'll try Neurontin. And then I usually talk to people about, you know, exercise. You know, there's data out there for acupuncture if they're inclined toward that type of intervention. And oftentimes they'll just get better with time, so encouraging just sticking it out and let's see if it gets better. Or just environmental changes, you know, 'What do you wear at night? What kind of sheets do you put on? Do you have a fan in your room,' you know, things like that."

"But nowadays, you know, all of them come in generic. So, they're a whole lot cheaper. But, see, in the years past they might have been $\$ 450$ a month; Femara was."

"I think most of them are weighing the wanting to get rid of the breast cancer. That's probably their primary motivation. I think secondarily they're thinking about issues of life expectancy and then I think it's quality of life."

"Most people, they're more scared about their breast cancer and so they want to be on these drugs."

"We refer them to fertility specialists. We have a clinic and they go over the issues too. And l've had a handful of people who've gone off tamoxifen and gotten pregnant."

"I think they use, patients tell me - I don't know if they're called 'chat rooms' or online, you know, kind of forums where people talk about their breast cancer diagnosis and their treatment. Patients get a lot of information from other breast cancer patients." 
Table I (Continued)

\begin{tabular}{|c|c|c|}
\hline Code & Definition & Exemplary quotes \\
\hline Informational materials & $\begin{array}{l}\text { Information that providers provide to } \\
\text { improve adherence (eg, information } \\
\text { from a study or a trial) }\end{array}$ & $\begin{array}{l}\text { "We sit them and explain it but they get educational materials too. Anything } \\
\text { that you can give for visual stimulation and also auditory. You know, } \\
\text { sometimes I think it's good to have a tape recording of your teaching of it and } \\
\text { then they can listen to that tape recording again." }\end{array}$ \\
\hline Interventions & $\begin{array}{l}\text { Intervention opportunities that } \\
\text { providers use to get patients to } \\
\text { initiate/adhere to ET }\end{array}$ & $\begin{array}{l}\text { "We run a clinical trial in the office where they can sign for this clinical trial } \\
\text { where they'll get text messages to remind them every day or I will set up their } \\
\text { iPhone for a daily reminder, you know, if they have that." }\end{array}$ \\
\hline Provider frustrations & $\begin{array}{l}\text { Provider-reported challenges for } \\
\text { influencing patient adherence }\end{array}$ & $\begin{array}{l}\text { "We have a lot of alternative people ... And they have such a mindset on the } \\
\text { alternative medications that they won't pick up what regular medicine does. } \\
\text { And you try to teach the fool out of it. But this is few and far between." }\end{array}$ \\
\hline No-brainer & $\begin{array}{l}\text { Discussion of the "obvious choice" } \\
\text { of ET }\end{array}$ & $\begin{array}{l}\text { "Most are pretty on board with it. I think, most (wouldn't you?), when we're } \\
\text { talking about invasive cancer, I think most are scared of their breast cancer, } \\
\text { and understandably so. So, I think most are willing to give a pill a try." }\end{array}$ \\
\hline Perceived (non) adherence & $\begin{array}{l}\text { Discussion of how big of a problem } \\
\text { nonadherence/persistence may be }\end{array}$ & "I would give you a guess [of nonadherence] would be I5\%-20\%." \\
\hline
\end{tabular}

Perceived (non) initiation Discussion of how big of a problem noninitiation may be for their patients

Perceived reasons

for (non) adherence

Perceived reasons

for (non) initiation

Prescribing decision making Includes which patients get what/ when, contraindications

Provider dismissiveness

Provider risk communication

Rare complications

Reminders

Research gap and recommendations
Risk of recurrence

Risk of side effects Shared decision making
Perceived research gaps and recommendations

Dismissing side effects and concerns of patients, discussion of misattribution of side effects with ET among patients

Includes discussion of risk perception, risk of side effects, and cancer risk scores

Discussion of rare complications to ET Discussion of risk of side effects

Discussion of risk of recurrence

Discussion of risk of side effects Description of shared decision-making process around ET
"Every once in a while you'll have somebody like that [noninitiator]. You know, l'm just talking about a very, very small population."

"I've even had people skip it for long plane rides and things. So, I can't come down too hard. But I do say 'Look, in general it's best to take all that'."

"[Some women are] looking at herbal things. And they don't like taking regular medications. And so, sometimes they're so holistic and so out there, you know, that they don't want to pick up a medication [ET] like that."

"Postmenopausal, we generally go with the orals and it's usually based on (several?) studies. So, most of our patients will be offered Arimidex first, since it was first and then depending on tolerance, they might get - in terms of an adjuvant setting, they might get switched over to one of the other hormonal therapies if they didn't tolerate Arimidex."

"And, admittedly, there is a population of folks out there who-you know, I don't want to in any way belittle their side effects but I think there's a population who are looking for some sort of side effect to attribute to the medication as an excuse not to take it."

"Usually you can sort of like draw a scale and say 'OK, here's the one thousandth chance that you're going to get uterine cancer from tamoxifen if that's what you're worried about. Here's, on the other side, your chance of dying from breast cancer will be changed by 100-1,000.' So I say 'Well, would you rather have $\$ 100$ or one dollar? Which is more?' And l'll say 'Well, then, this is what you want to do.' And there's usually some way you can get through and make sense of it."

"We know there's endometrial cancer, blood clots."

"I tell them to put it in a place so that when they do their repetitive daily action, such as brushing their teeth or blow drying their hair or showering or whatever, they will see it and take it. Or use a pill box with Monday through Friday kind of thing."

"I guess the only thing that I'm hopeful for, and I think there are some studies ongoing, are trying to understand who we can predict to be the patients who are going to be poorly tolerant of the aromatase inhibitors; either clinical factors or even, you know, sniff analysis and pharmaco-genomic studies will really help us, our pharmaco-genomic studies will really help us understand who may be less tolerant so we can go ahead and start intervention programs ahead of time." 'YYou can calculate the risk of recurrence. The words 'high,' 'low risk,' you know, mean many different things."

"You know, most people want to know what the side effects and risks are." "I mean I think modern breast cancer therapy is very much a collaborative doctor-patient situation for most of us. I mean, you know, I think it's pretty rare for somebody to sort of give me the impression that she wanted to go ahead and proceed with therapy, take the prescription and then not do it." 
Table I (Continued)

\begin{tabular}{|c|c|c|}
\hline Code & Definition & Exemplary quotes \\
\hline Side effects: cognition & $\begin{array}{l}\text { Description of patient report of } \\
\text { cognitive side effects, includes things } \\
\text { such as cognitive lapses, due to ET }\end{array}$ & "I've had a few patients that they feel like they're $[\ldots]$ in a fog." \\
\hline Side effects: cosmetic & $\begin{array}{l}\text { Description of patient experiences } \\
\text { with hair loss and other cosmetic } \\
\text { issues due to ET }\end{array}$ & $\begin{array}{l}\text { "I would say cosmetic symptoms ... changes in hair pattern. Those are always } \\
\text { disturbing." }\end{array}$ \\
\hline Side effects: hot flashes & $\begin{array}{l}\text { Description of experiences with hot } \\
\text { flashes due to ET }\end{array}$ & $\begin{array}{l}\text { "So, for the tamoxifen, you know, for the younger women, hot flashes is } \\
\text { probably the number one." }\end{array}$ \\
\hline Side effects: joint/bone pain & $\begin{array}{l}\text { Description of experiences with } \\
\text { joint/bone due to ET; also includes } \\
\text { leg cramps }\end{array}$ & $\begin{array}{l}\text { "So, they tend to have more trouble. And then the joint pains is the other } \\
\text { main symptomatic issue. And the classic, from my reading of the literature and } \\
\text { my experience, what I think is the classic is the hand, you know, swelling and } \\
\text { pain, stiffness in the hands." }\end{array}$ \\
\hline Side effects: mood & $\begin{array}{l}\text { Description of experiences with } \\
\text { mood swings/mental health issues } \\
\text { associated by patients with ET; } \\
\text { also includes leg cramps }\end{array}$ & $\begin{array}{l}\text { "So, and actually mood disturbance too, you know, feeling irritable. I think that } \\
\text { probably is real to some extent for some folks." }\end{array}$ \\
\hline Side effects: other & $\begin{array}{l}\text { Discussion of other side effects } \\
\text { attributed by patients to ET }\end{array}$ & $\begin{array}{l}\text { "I've had a few patients that they feel like they're nauseous while taking the } \\
\text { medicine." }\end{array}$ \\
\hline Side effects: overwhelming & $\begin{array}{l}\text { Instances when patients explicitly } \\
\text { discuss side effects as "overwhelming" } \\
\text { or quit ET because the side effects } \\
\text { are overwhelming }\end{array}$ & $\begin{array}{l}\text { "Other patients have tried it for a brief period of time and just felt like they } \\
\text { don't tolerate it. They have joint aches and pains that are just unbearable and } \\
\text { tamoxifen wouldn't be an option for them due to previous clotting or stroke } \\
\text { history after trying all the Als." }\end{array}$ \\
\hline Side effects: sexual function & $\begin{array}{l}\text { Description of patient experiences } \\
\text { with sexual function due to ET }\end{array}$ & $\begin{array}{l}\text { "No. It's more about - the other big issue on both of them is obviously loss } \\
\text { of libido, vaginal dryness, lack of interest, and then how to deal with that. } \\
\text { So, I generally have a sexuality conversation with all the patients and ask them } \\
\text { how that's going and if they're having any symptoms or any problems." }\end{array}$ \\
\hline Side effects: weight gain & $\begin{array}{l}\text { Description of patient experiences } \\
\text { with weight gain due to ET }\end{array}$ & $\begin{array}{l}\text { "Sometimes we'll see some weight gain. The literature doesn't suggest that } \\
\text { they're going to have weight gain, but I definitely see it." }\end{array}$ \\
\hline
\end{tabular}

Abbreviations: Als, aromatase inhibitors; ET, endocrine therapy; DCIS, ductal carcinoma in situ; ER, estrogen receptor; PR, progesterone receptor.

Verbal informed consent was obtained from all individual participants included in the study. This study was approved by the University of North Carolina at Chapel Hill Institutional Review Board.

\section{Results}

Oncology providers who were interviewed were primarily medical doctors (70\%), although $20 \%$ were nurse practitioners, and $10 \%$ were physician assistants (Table 2). The average age of providers interviewed was 48 years, with an average of 17.3 years since their medical training. Providers reported seeing $\sim 33$ breast cancer patients per week, and reported that just over two-thirds of their practices were breast cancer patients. We would expect approximately threequarters of these patients to be HR+ and eligible for ET. ${ }^{11}$ Most practiced in a suburban (55\%) or urban (40\%) setting, whereas only $5 \%$ practiced in a rural setting. Just over half reported practicing in an academic medical center.

The salience of specific codes, in terms of both overall frequency of mention and the proportion of providers who mentioned each code, is reported in Table 3. For example, $95 \%$ or more of providers mentioned the following in their interviews: coping strategies; perceived noninitiation; perceived nonadherence; perceived reasons for nonadherence; prescribing decision making; provider risk communication; hot flashes; and joint pain. These topics were among the most frequently mentioned codes across all transcripts. In the following pages, the overarching themes from

Table 2 Descriptive characteristics of oncology providers interviewed

\begin{tabular}{ll}
\hline Characteristics & Mean, \% \\
\hline Age (years) & 47.8 \\
Provider role (\%) & \\
Medical doctor & 70.0 \\
Nurse practitioner & 20.0 \\
Physicians assistant & 10.0 \\
Years since training & 17.3 \\
Patient mix (\% breast cancer patients) & 68.0 \\
Patient load (per week) & 32.9 \\
Practice setting (\%) & \\
$\quad$ Urban & 40.0 \\
Rural & 5.0 \\
Suburban & 55.0 \\
Academic (vs nonacademic) (\%) & 55.0 \\
\hline
\end{tabular}


Table 3 Salience of coded concepts in interview data

\begin{tabular}{|c|c|c|}
\hline Code & Code frequency & $\%$ Providers who mentioned code \\
\hline Coping & 94 & 100 \\
\hline Perceived (non) initiation & 52 & 100 \\
\hline Perceived reasons for (non) adherence & 59 & 100 \\
\hline Prescribing decision making & 90 & 100 \\
\hline Provider risk communication & 77 & 100 \\
\hline Side effects: hot flashes & 40 & 100 \\
\hline Perceived (non) adherence & 36 & 95 \\
\hline Side effects: joint/bone pain & 42 & 95 \\
\hline Risk of recurrence & 41 & 90 \\
\hline Perceived reasons for (non) initiation & 60 & 80 \\
\hline Compelling arguments & 17 & 75 \\
\hline Risk of side effects & 36 & 75 \\
\hline Side effects: other & 20 & 55 \\
\hline Side effects: sexual function & 14 & 50 \\
\hline Double-edged sword & 14 & 45 \\
\hline Informational materials & 15 & 45 \\
\hline Rare complications & 9 & 45 \\
\hline Side effects: overwhelming & 9 & 45 \\
\hline Research gap and recommendations & 10 & 40 \\
\hline Information seeking & 9 & 35 \\
\hline Beliefs in line with behavior & 9 & 30 \\
\hline Fertility & 6 & 30 \\
\hline Reminders & 9 & 30 \\
\hline Side effects: mood & 7 & 30 \\
\hline Age-based decision making & 8 & 25 \\
\hline Provider frustrations & 5 & 25 \\
\hline Provider dismissiveness & 9 & 20 \\
\hline Shared decision making & 6 & 20 \\
\hline Side effects: cosmetic & 4 & 20 \\
\hline Side effects: cognition & 4 & 15 \\
\hline Fear & 2 & 10 \\
\hline Interventions & 3 & 10 \\
\hline No-brainer & 2 & 10 \\
\hline Side effects: weight gain & 3 & 10 \\
\hline Comorbid conditions & 2 & 5 \\
\hline
\end{tabular}

these in-depth interviews, as well as illustrative verbatim quotes, are presented (see "Methods" section).

\section{Providers recommend ET to virtually all eligible patients except those with medical contraindications, significant comorbidities, or other serious risk factors}

According to the providers interviewed, the majority of their breast cancer patients $(\sim 70 \%)$ are estrogen or progesterone receptor-positive. Providers recommended ET to virtually all of their HR+ patients except in the rare instances of medical contraindications. Some of the providers described it this way:

The majority of those I see who are hormone receptor-positive are going to be on some kind of endocrine therapy ... [I always recommend it] because it's safer than aspirin.
I would say that maybe once or twice a year, [I might not recommend ET] to somebody who had a compelling reason to be on ET but who had risk factors from both the AI and Tamoxifen that outweighed the benefits. So imagine somebody who had a high enough risk of breast cancer that you wanted to give her ET, but she had already had three bone fractures from osteoporosis and a history of multiple blood clots. Essentially you'd have no options.

Physicians reported that they have the first conversation about ET with all eligible patients soon after diagnosis as part of the projected overall treatment plan. This way, ET will be viewed by the patients as the next step in - and an integral part of - the treatment regimen. Later, after the patient has had surgery and completed chemotherapy and/ or radiation therapy, the physicians discuss the intricacies of 
ET in greater detail closer to the time the patient is ready to take ET. As these physicians explained:

We end up talking about it [ET] several times. ... I mention it in the very first consultation ... as part of the whole package. ... We talk about, now that this cancer is out of their body, what's the likelihood that they might have a recurrence and how recurrence can happen even if the doctor resected it all. ... We talk about the biology of recurrence and make sure they understand that we're talking about reducing a risk of something happening, but that we don't know that that's going to happen to them. I set the stage of what's the situation we're in to help people weigh the risks and benefits. ... We talk about estrogen receptors, and we say 'OK, for your cancer, we need to use this element and that element, and then, down the road, we're going to use this anti-estrogen. We're going to talk about that later, but it's important that you know about it.'

I always tell my patients the pathology report is one of the most important pieces of information. ... We'll actually go through it, and I'll draw the receptors on a breast cancer cell for patients so they understand how their cancer grows. Then I draw an X through it and say, 'This is what the drug is going to do,' to really hit them over the head with how important this therapy is. If they're estrogen receptor-positive, we're having that conversation at their very first visit. Whether they remember it is another question ... but I try to make certain it's a part of the treatment algorithm.

What I really try to instill in them upfront is 'ET is really important for you in terms of risk reduction. This is not like a 1 percent reduction of risk. This could be upwards of 10 or 15 percent risk reduction.' I always give both benefits and risks, and I try to indicate to the patient that this is a very safe therapy, but that some what I would call nuisance side effects many times make the drug such a nuisance that it's intolerable.

\section{Providers consider ET an integral part of active treatment, the most compelling argument for which is its value in reducing the risk of recurrence}

The providers we interviewed said that they present each patient's individual risk of recurrence to her and the potential ET has of reducing that risk. Most often, this information is presented in "absolute risk" terms because they have found that to be the most understandable way for patients to process the treatment decision. As these providers described it:
I'll give them the risk-benefits percentages. In general, endocrine therapy will decrease the risk of the cancer coming back by 40 percent. So I will talk to them about that and about how the risk factors of the medicine may be less than 5 percent. If they still want to make the choice not to take it, it's their choice. I'll respect that. But I usually go over the benefit of the medicine a few times to make sure they really understand. ... I've learned to use the analogy of 'There are 100 women [just like you] sitting in the room, and 40 of them are going to have their cancer come back. And if those 40 take [ET] then only 20 to 24 of them will have it come back.' They can actually envision people sitting in a room.

Since Americans are notoriously terrible at math, I use absolute numbers. I'll say, 'If I had 100 women like you in a room and we did this and that, this is what would happen. And if [these 100 women] took these pills, we would save this many people in that room and prevent a lot of relapses.'

Some providers described a more nuanced communication and decision-making process around risk-benefit trade-offs:

In the adjuvant setting, it is all about weighing the risks and benefits. So it's very important for the oncologist to try to accurately quantify the risk of recurrent breast cancer if we do nothing in terms of therapy and also to make sure the patient understands what we are talking about when we talk about recurrence. We're not just talking about another lump in the breast. We're talking about the likelihood that there's a rogue cell that could come back as a metastasis in your liver. So if we take no therapy at all, is that risk 40 percent or 4 percent? And then your net gain from using ET is weighed depending on how high-risk your disease is to begin with. ... I try to give patients an adequate sense of what we are gaining and what we are losing if we take an ET. For example, if someone is 90 or 75 with a multitude of other medical problems and has a stage one, small node-negative, estrogen receptor positive breast cancer and you crunch those numbers, the risk of a recurrence over the next five to 10 years might be something like 15 percent. Her risk of dying from something else on her medicine list might be higher than that. Adjuvant endocrine therapy probably would reduce her risk of recurrence by about 5 or 6 percentage points. You and she might decide that's simply not worth having to take another pill or worth the risk of fracture or hot flashes. ... You have this very balanced discussion and say, 'Well, you know, 2 percent of women have blood clots and 1 percent have uterine cancer, and I think your net gain from Tamoxifen might be $3 \frac{1}{2} 2$ percent.' And 
they might say, 'OK, thank you very much. I'm not going to take that,'... because it might be sort of a gray area where the odds don't [justify it]. ... We joke that ... the length of the consultation increases as the size of the tumor decreases. It's easy if there's a high-risk cancer and you and the patient both want to treat it with everything.

All of the providers reported that they emphasize to their patients the potential long-term value of ET. In fact, many cited research that has shown ET to be more important than chemotherapy in preventing recurrences. Some of the providers elaborated on the importance of ET relative to other treatments:

I tell patients that ET is more important than chemotherapy because the absolute benefit you derive from chemotherapy is anywhere from 2 to 7 percent, depending on the biology of your cancer, whereas Tamoxifen reduces your risk of recurrence usually on the order of 40 to 50 percent, and it reduces the risk of contralateral new breast cancer by 50 percent.

[I tell the patient] that proportional benefits [of ET] [are] higher than you would actually receive from chemotherapy, which is one-third proportional reduction. I remember having multiple conversations with a patient who preferred chemotherapy over endocrine therapy. I tried to explain that the margin of benefit is actually lower for the chemo than endocrine therapy alone. Many patients' risks are so high we need to combine everything we possibly can: chemotherapy, endocrine therapy, and the like. But when you're really talking about the proportional benefit, the endocrine therapy has a much higher proportional benefit than chemo itself.

\section{Providers base their decision of which ET to use on the patient's menopausal status, percent of estrogen positivity, comorbidities, and risk factors}

Providers noted they first take into account menopausal status to determine which ET to provide. Generally speaking, premenopausal, HR+ females are candidates for tamoxifen, while postmenopausal females are candidates for an AI. Those who are premenopausal and later become postmenopausal use a combination of the two. Other factors that affect their choice of therapies are females' comorbid medical conditions and risk factors. For example, females with a history or predisposition toward thrombosis would not be good candidates for tamoxifen, while an AI would not be advisable for females with severe osteoporosis. In the rare cases where females have risk factors for both kinds of therapy, or who are elderly and frail, providers explained they may not recommend ET at all if their degree of cancer risk is low. Generally, postmenopausal patients receive 5 years of treatment with an AI. Since the Atlas study, the trend among these providers regarding tamoxifen is to move toward 10 years of treatment. ${ }^{12}$ Some of the providers explained their prescribing decisions this way:

The main determinant is menopausal status. In premenopausal women, I don't use an AI. With a patient who has osteoporosis, I may not start with an AI. Conversely, with a patient with a deep vein thrombosis (DVT) or thrombolytic history, I wouldn't like to start with Tamoxifen. So those are the main comorbidities that I take into account. Patients who have a lot of trouble with musculoskeletal systems are sometimes leery of AIs, at least initially.

In the pre-menopausal category, my therapy of choice is Tamoxifen, unless a patient has a predisposition for blood clotting, history of DVTs ... or some other medical reason to steer clear of Tamoxifen based on their clotting risk or endometrial pathology. If I have a patient with a medical contra-indication to Tamoxifen, I pursue ovarian oppression ... so I can medically place a patient in menopause. ... And then I would prescribe an aromatase inhibitor with ovarian suppression because I've made them chemically post-menopausal. Then, nine times out of 10 , I start with an aromatase inhibitor. ... I've followed the lead of five years of the aromatase inhibitor. You could select a steroidal (Aromasin or Exemestane) or non-steroidal (Anastrozole or Arimidex). The head-to-head comparison showed no long-term difference in efficacy, [but] there were differences in the toxicity profiles. ... The steroidal had a slightly reduced risk of osteoporosis. My aromatase inhibitor of choice is Letrozole upfront ... based on how I was trained and the first drugs that came onto the market. ... But I'm keen to switch patients over to Aromasin if they have arthralgias and myalgias, because I find that some patients who don't tolerate Letrozole or Arimidex will very likely tolerate Aromasin.

\section{Providers believed that noninitiation of ET is rarely a problem; in fact, they reported that the vast majority of their patients are extremely receptive to the concept of taking ET}

All of the providers we interviewed indicated that the vast majority of their patients (approximately $90 \%-95 \%$ ) are very 
receptive to the idea of taking ET. They said that only a few choose not to initiate this therapy. According to those interviewed, their patients generally view ET positively because of the comfort and reassurance of being able to do something else to prevent recurrence after chemo and/or radiation. As these providers explained:

I find that when patients are taking that pill, they feel more reassured they're fighting their cancer every day. ... It's a bit of reassurance that they're doing everything that they possibly can to prevent breast cancer recurrence.

I think they come highly motivated. This is the scare of their life for many people, and they've all seen a friend or family member die [of cancer]. Most women come to it saying, 'What can I do?' And frankly, if they don't have to have chemo or if they've already gotten through chemo, endocrine therapy is relatively easy. It's not a sell job in most cases.

Providers reported that they believe that the handful of patients who reject ET from the outset generally do so because of: fear of side effects and risks; negative feedback about ET from friends or on the Internet; reluctance to use "any" drug long-term, especially one that would impact their endocrine system; incompatible fertility intentions; distrust or skepticism of Western medicine; preponderance toward holistic or alternative medicine to prevent recurrence in lieu of ET; or an inability to function adequately or organize their lives. Almost all of the providers reported that in the current era, financial considerations are rarely a barrier because of the availability of generics. Some of the providers painted a picture of their patients who have refused to try ET in the following ways:

I certainly do have a handful of patients who [do not initiate] due to social constraints, financial constraints, or their own inability to organize their lives. Financial constraints have become much less of a barrier [over time] in my clinic.

Usually they've heard something terrible about the medicine from either the Internet or from people they know who have taken the medicine, or they just have the feeling that they don't want to block hormones in their body. They just have a constitutional idea that they're not going to do it no matter what. And especially with Tamoxifen, they've heard about a lot of side effects, so they decide not to take it.

Sometimes patients don't like taking medications at all or they read on the Internet that it's better to take Ginkgo biloba than to take Western medicines. ... And some patients make a thoughtful decision that they just don't want to. If they're 85 years old, they may say, ... 'I just decided I don't want to add another medicine to my pile.'

Sometimes they come with the preexisting decision that they're not going to do this. In those cases I feel like my job is to present them with the information about why we use this medication and why and how much it might help and then to allow them to make their own decision. I think as long as somebody's competent and they've heard and understood, it's not really my job to beat them up. ... But if the patient has an extremely high-risk cancer and it's strongly ER positive, you know she needs to take this drug. Then I do try to motivate [her]. Typically what we do is we have them give it a try and then have a short interval follow-up. And I try to see what the barriers are. We try to get at the 'what can we work on to make this drug work for you?' Sometimes you get there, and sometimes you don't.

\section{Providers believed that adherence and persistence were much more problematic with ET than noninitiation, mostly because of intolerable, unresolvable side effects}

According to the providers, some of their patients who were motivated to take ET discontinued it because of intolerable and unmanageable side effects that severely impacted the quality of their lives. Providers reported perceived harms of tamoxifen to be: hot flashes that interfere with sleep and daily life (the most common complaint); weight gain (perceived by females, though not supported by research); depression; loss of libido and loss of sexual interest; vaginal dryness, vaginal atrophy, and perineal pain; and, much less often, frequent urinary tract infections (UTIs), blood clots, and other rare events.

Providers reported the perceived harms of AIs to be: arthralgia and myalgia, especially joint pain (the No 1 complaint); weakening of the bones (osteoporosis); and less often, loss of libido, loss of sexual interest, vaginal dryness, atrophy, and perineal pain.

If, in spite of efforts to reduce them, severe side effects continue to impact quality of life, some females choose to discontinue ET, and the providers typically respect that decision. One provider described her experience with nonadherers this way:

Some people have had such severe joint pain and their risk has been low enough and they've tried one or two drugs. I had an elderly woman who said, 'I can't live my life like this. I just can't do this.' You have to be respectful ... and 
they're equally respectful. They appreciate that you're making your best medical recommendation. It is all about balance and quality of life.

\section{Providers reported using several pharmacological and nonpharmacological approaches to help females deal with the side effects of ET and better support females taking ET}

Providers help patients manage ET side effects (eg, hot flashes and joint pain) with a variety of nonpharmacological and pharmacological methods. To deal with hot flashes, providers first recommend females try layering clothes, using fans, avoiding caffeine and spicy foods, reducing their alcohol intake, pursuing a healthy diet and exercise, and using progressive relaxation, other mindfulness techniques, and acupuncture. If necessary, they also prescribe medications such as venlafaxine, gabapentin or clonidine. Most of the providers also screen for depression. For vaginal dryness, they typically recommend lubricants or lidocaine for intercourse.

Often there is nothing providers can do to make the joint pain tolerable other than switching to another drug in the class to see whether it is better tolerated. Providers try to lessen the joint pain by recommending over the counter pain relievers, exercise, vitamin D, yoga, tai chi, and acupuncture, with varying success. Some report success with acupuncture, although providers noted that acupuncture can be expensive and may not be covered by insurance. One provider said her hospital offered free acupuncture. Providers describe these strategies:

We spend the bulk of the survivorship care for people who are on anti-estrogen therapy or who have gone through chemotherapy-induced menopause managing side effects. There's a whole litany of things you can do for hot flashes. We are screening for depression and anxiety at every visit. We're checking vitamin D levels, and we spend a lot of time talking about arthralgia. The nurse navigators field a lot of calls about symptoms as well and try to strategize with the women - everything from common sense, 'wear layers,' to vitamin E to gabapentin.

The AI's are tough. It's the joint pains and muscle aches. What the patients basically say is 'I feel like I'm a hundred years old.' It's worse in the morning for a lot of these patients. There are some studies that show yoga can be helpful. ... There's some minor data that people with lower vitamin $\mathrm{D}$ [have more joint pain]. ... Adding vitamin D can make it tolerable, and that's what you're looking for. If it's that uncomfortable, you can switch to another AI, which they may tolerate better. ... I think a small number actually stop ... though more may become more erratic in taking it or not. ... Their fear of the risk of recurrence is a big motivator.

\section{Discussion}

Because ET is a vital component of active treatment for $\mathrm{HR}+$ females and ET initiation, adherence, and persistence are known to be suboptimal, the perspectives of providers managing these patients are critical to understanding the challenges and opportunities involved in optimizing care for this patient population. Our in-depth interviews suggest that providers recommend ET in a consistent manner, and while these providers believed that ET noninitiation is rare, ET nonadherence was cited as a major source of concern for the management of $\mathrm{HR}+$ breast cancer patients. In particular, persistent side effects were believed to be a major contributor to nonadherence, and providers expressed encountering significant challenges managing these side effects due to heterogeneity in patient experience, and limited options and evidence for successfully managing side effects. Thus, while many of the other barriers may be important, side effect management in particular should be a priority for research into improved side effect management and for health services/comparative effectiveness research to identify best practices and disseminate this knowledge to providers. Overall, providers discussed the important role of risk communication as a motivating factor for both ET initiation and adherence. In particular, providers discussed the advantages for discussing risk in absolute rather than relative terms with patients.

Nonadherence was primarily associated with persistent and unmanageable side effects from ET, primarily hot flashes, night sweats, and joint pain. This aligns with literature associating high side effect burden with ET nonadherence., ${ }^{2,13,14}$ Other barriers associated with ET adherence have been presented in the literature, such as minority race/ethnicity, ${ }^{13}$ cost, poor patient-provider communication, and others. ${ }^{2,14,15}$ However, these factors were discussed less often by providers, perhaps because side effects are the most significant barriers that patients experience and discuss with their providers. ${ }^{15}$ Oncology training and practice tend to emphasize disease treatment guidelines, not guidelines for management of side effects of treatment, which represents an understudied area that is important to improving patient outcomes and quality of life. 
Most providers felt that the majority of their patients were willing to try ET and that noninitiation was rarely a concern. They believed most patients agreed with the premise of ET in terms of its efficacy in reducing recurrence risk. Patient data from other settings, however, suggest that noninitiation may be prevalent, most often for females of lower socioeconomic ${ }^{16}$ status or minority race or older age. ${ }^{14,17}$ Providers from this study associated noninitiation with patient distrust of conventional medicine. Patientreported reasons for noninitiation should be further explored in future work. In some cases, providers may not even know that their patients never initiated therapy. Accordingly, research is also needed that explores patient-provider communication about medication taking behavior after prescriptions are written.

Our data point to several takeaways for practicing oncology providers, including: to more effectively communicate ET risks and benefits, particularly emphasizing the role of ET in reducing risk of recurrence, and help patients to adequately cope with and tolerate ET in light of significant and sometimes debilitating side effects. These suggestions require thoughtful implementation in order to be realized most optimally, since 1) the clinic environment can be quite busy and individual visits with oncology care providers are shorter than ideal; 2) a one-size-fits-all strategy will not be appropriate for risk communication or for side effect management; and 3) messaging must be continual and consistent to help support ET adherence and persistence for 5-10 years, as recommended. Strategies to address these challenges may include using more lay or peer navigators in the clinic (who can afford to spend more time with each individual patient), ${ }^{18,19}$ tailoring risk communication ${ }^{20}$ and side effects management to better fit with the individual patient's experience, ${ }^{21,22}$ and consolidating evidence-based information about effective management of ET-related side effects, including pharmacological and nonpharmacological options. $^{22}$

While previous studies have examined the barriers and facilitators for ET use among certain subgroups of breast cancer patients, ${ }^{23,24}$ this study, to our knowledge, is the first qualitative study to examine providers' perspectives on ET prescribing, initiation, and adherence. These perspectives are essential in understanding the key barriers and facilitators for the use of ET among breast cancer patients. Furthermore, to promote shared decision making regarding ET use, it is essential that both patients and providers have a shared understanding about these key barriers and facilitators of ET use. Patients in prior studies ${ }^{23,24}$ and providers in our study primarily discussed suboptimal symptom management as the main barrier for ET use. But evidence suggests that females often do not feel empowered to discuss symptom management with their providers and even when they do raise the issue, symptoms are not always easily addressible. ${ }^{23}$ Furthermore, patients have previously reported not knowing that missing ET doses could reduce the medication's efficacy. ${ }^{23}$ So, while both patients and providers agree that symptom management is important, there seems to be a disconnect in the delivery of symptom management strategies to patients in need.

\section{Limitations}

Some limitations of this study require careful acknowledgement. While we interviewed providers across geographic regions, health care settings, and patient-care roles, these provider perspectives may not be generalizable to providers who did not opt to participate in the study. It is possible that providers we interviewed who felt that certain issues were not relevant (eg, noninitiation) may have simply been reflecting the experiences of their own panel of patients, which may not be representative of all patients' and providers' experiences. Despite these limitations, we believe our internal validity was high; we spent considerable time developing and revisiting the codebook, discussing code application in detail when questions emerged during the coding process, and assessing code consistency and reliability on a subset of transcripts. Additionally, we achieved saturation of themes in our 20 interviews.

\section{Conclusion}

This study identifies priority areas for engaging providers to help improve ET adherence among females with HR+ breast cancer. Specifically, side effects from ET treatments represent the dominant factor in nonadherence, and evidence suggests that some patients experience considerable difficulty managing such symptoms. Efforts to manage the most troublesome symptoms may fail because of the limited number of effective management strategies currently available. Efforts to improve adherence should focus on providerdirected strategies to prepare patients for and help minimize side effects, empower patients to seek help when side effects become unmanageable, and ensure that both clinicians and patients are educated about and continue to discuss optimal side effect management as problems emerge. This finding has important implications for the management of $\mathrm{HR}+$ breast cancer patients, especially as evidence points to ET regimens that extend beyond 5 years. 


\section{Acknowledgments}

This research was funded by the American Cancer Society (ACS) Mentored Research Scholar Grant (MRSG-13-15701-CPPB: "Improving Endocrine Therapy Utilization in Racially Diverse Populations"). SBW was also supported as a faculty trainee through the Carolina Community Network Center to Reduce Cancer Health Disparities (CCN II), funded by the National Cancer Institute's Center to Reduce Cancer Health Disparities through its Community Network Program Centers (U54-CA153602). JAE and MCR were supported through UNC Lineberger, Cancer Control Education Program (R25 CA57726). During the conduct of this research, KRH received funding from a career development award through the Building Interdisciplinary Careers in Womens' Health (BIRCWH) program of the National Institutes of Health (5K12HD001441-12). CEG was partially supported by the Eunice Kennedy Shriver National Institute of Child Health and Human Development (K24-HD06920).

\section{Disclosure}

The authors report no conflicts of interest in this work.

\section{References}

1. Carlson RW, Hudis CA, Pritchard KI; National Comprehensive Cancer Network; Breast Cancer Clinical Practice Guidelines in Oncology; American Society of Clinical Oncology Technology Assessment on the Use of Aromatase Inhibitors; St Gallen International Expert Consensus on the Primary Therapy of Early Breast Cancer. Adjuvant endocrine therapy in hormone receptor-positive postmenopausal breast cancer: evolution of NCCN, ASCO, and St Gallen recommendations. J Natl Compr Canc Netw. 2006;4(10):971-979.

2. Murphy CC, Bartholomew LK, Carpentier MY, Bluethmann SM, Vernon SW. Adherence to adjuvant hormonal therapy among breast cancer survivors in clinical practice: a systematic review. Breast Cancer Res Treat. 2012;134(2):459-478. doi: 10.1007/s10549-012-2114-5. Epub 2012 Jun 12.

3. Cluze C, Rey D, Huiart L, et al. Adjuvant endocrine therapy with tamoxifen in young women with breast cancer: determinants of interruptions vary over time. Ann Oncol. 2012;23:882-890.

4. Dent SF, Gaspo R, Kissner M, Pritchard KI. Aromatase inhibitor therapy: toxicities and management strategies in the treatment of postmenopausal women with hormone-sensitive early breast cancer. Breast Cancer Res Treat. 2011;126:295-310.

5. Oberguggenberger A, Hubalek M, Sztankay M, et al. Is the toxicity of adjuvant aromatase inhibitor therapy underestimated? Complementary information from patient-reported outcomes (PROs). Breast Cancer Res Treat. 2011;128:553-561.

6. Bhosle MJ. Outcomes Associated with Adjuvant Hormonal Therapy: Are There Any Differences Between Black and White Women with Primary Breast Cancer? Ann Arbor: ProQuest Information \& Learning; 2008.

7. Files JA, Ko MG, Pruthi S. Managing aromatase inhibitors in breast cancer survivors: not just for oncologists. Mayo Clin Proc. 2010;85:560-566.
8. Smith SL, Wai ES, Alexander C, Singh-Carlson S. Caring for survivors of breast cancer: perspective of the primary care physician. Curr Oncol. 2011;18:e218-e226.

9. Goss PE, Ingle JN, Pritchard KI, et al. Extending Aromatase-Inhibitor Adjuvant Therapy to 10 Years. N Engl J Med. 2016 Jun 5. [Epub ahead of print].

10. Ward JH. Duration of adjuvant endocrine therapy of breast cancer: how much is enough? Curr Opin Obstet Gynecol. 2010;22:51-55.

11. American Cancer Society. Breast Cancer Overview. American Cancer Society Web site; Last Revised January 15, 2016. Available from: http:// www.cancer.org/cancer/breastcancer/detailedguide/breast-cancertreating-hormone-therapy. Accessed February 2, 2016.

12. Davies C, Pan H, Godwin J, et al. Adjuvant Tamoxifen: Longer Against Shorter (ATLAS) Collaborative Group; Long-term effects of continuing adjuvant tamoxifen to 10 years versus stopping at 5 years after diagnosis of oestrogen receptor-positive breast cancer: ATLAS, a randomised trial. Lancet. 2013;381(9869):805-816.

13. Kahn KL, Schneider EC, Malin JL, Adams JL, Epstein AM. Patient centered experiences in breast cancer: predicting long-term adherence to tamoxifen use. Med Care. 2007;45(5):431-439.

14. Roberts MC, Wheeler SB, Reeder-Hayes K. Racial/Ethnic and socioeconomic disparities in endocrine therapy adherence in breast cancer: a systematic review. Am J Public Health. 2015;105(Suppl 3):e4-e15. doi: 10.2105/AJPH.2014.302490. Epub 2015 Apr 23.

15. Pellegrini I, Sarradon-Eck A, Soussan PB, et al. Women's perceptions and experience of adjuvant tamoxifen therapy account for their adherence: breast cancer patients' point of view. Psychooncology. 2010;19: 472-479.

16. Wheeler SB, Kohler RE, Reeder-Hayes KE, et al. Endocrine therapy initiation among Medicaid-insured breast cancer survivors with hormone receptor-positive tumors. J Cancer Surviv. 2014;8(4):603-610. doi: 10.1007/s11764-014-0365-3. Epub 2014 May 28.

17. Riley GF, Warren JL, Harlan LC, Blackwell SA. Endocrine therapy use among elderly hormone receptor-positive breast cancer patients enrolled in Medicare Part D. Medicare Medicaid Res Rev. 2011;1(4).

18. Freund KM, Battaglia TA, Calhoun E, et al. National Cancer Institute Patient Navigation Research Program: methods, protocol, and measures. Cancer. 2008;113:3391-3399.

19. Ko NY, Darnell JS, Calhoun E, et al. Can patient navigation improve receipt of recommended breast cancer care? Evidence from the National Patient Navigation Research Program. J Clin Oncol. 2014;32: 2758-2764.

20. Burton M, Collins KA, Lifford KJ, et al. The information and decision support needs of older women ( $>75 \mathrm{yrs}$ ) facing treatment choices for breast cancer: a qualitative study. Psychooncology. 2015;24:878-884.

21. Lubberding S, van Uden-Kraan CF, Te Velde EA, Cuijpers P, Leemans CR, Verdonck-de Leeuw IM. Improving access to supportive cancer care through an eHealth application: a qualitative needs assessment among cancer survivors. J Clin Nurs. 2015;24:1367-1379.

22. Cella D, Fallowfield LJ. Recognition and management of treatmentrelated side effects for breast cancer patients receiving adjuvant endocrine therapy. Breast Cancer Res Treat. 2008;107:167-180.

23. Harrow A, Dryden R, McCowan C, et al. A hard pill to swallow: a qualitative study of women's experiences of adjuvant endocrine therapy for breast cancer. BMJ Open. 2014;4(6):e005285.

24. Wheeler S, Roberts MC, Bloom D, et al. Understanding barriers and facilitators to endocrine therapy use: a qualitative study of racially diverse breast cancer patients and providers. Paper presented at: Academy Health, Annual Research Meeting; June 2014; San Diego, CA. 
Patient Preference and Adherence

Dovepress

\section{Publish your work in this journal}

Patient Preference and Adherence is an international, peer-reviewed, open access journal that focuses on the growing importance of patient preference and adherence throughout the therapeutic continuum. Patient satisfaction, acceptability, quality of life, compliance, persistence and their role in developing new therapeutic modalities and compounds to optimize

Submit your manuscript here: http://www.dovepress.com/patient-preference-and-adherence-journa clinical outcomes for existing disease states are major areas of interest for the journal. This journal has been accepted for indexing on PubMed Central. The manuscript management system is completely online and includes a very quick and fair peer-review system, which is all easy to use. Visit http://www. dovepress.com/testimonials.php to read real quotes from published authors. 